\title{
Review of: "Significant modulations of linc001128 and linc0938 with miR-24-3p and miR-30c-5p in Parkinson disease"
}

Xuezhong Li

Potential competing interests: The author(s) declared that no potential competing interests exist.

This article has made a well study on significant modulations of linc001128 and linc0938. Two novel IncRNAs as the biomarker of PD diagnosis and treatment are revealed for the first time $]$ this work is of great innovative value. I feel this is a reasonably good study that might be published as it is 